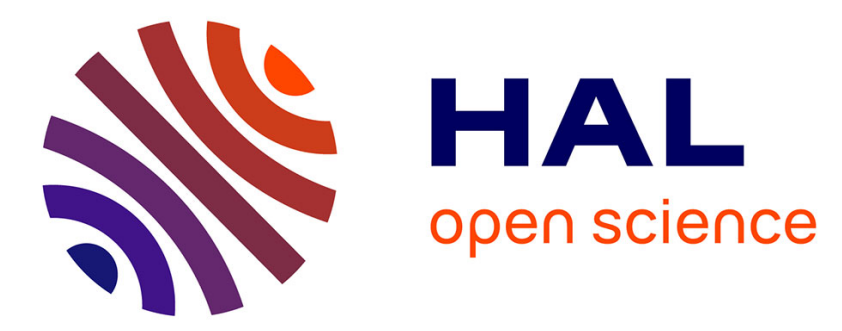

\title{
Differentiating sigma-fields for Gaussian and shifted Gaussian processes
}

\author{
Sébastien Darses, Ivan Nourdin, Giovanni Peccati
}

\section{To cite this version:}

Sébastien Darses, Ivan Nourdin, Giovanni Peccati. Differentiating sigma-fields for Gaussian and shifted Gaussian processes. 2007. hal-00128162

\section{HAL Id: hal-00128162 \\ https://hal.science/hal-00128162}

Preprint submitted on 31 Jan 2007

HAL is a multi-disciplinary open access archive for the deposit and dissemination of scientific research documents, whether they are published or not. The documents may come from teaching and research institutions in France or abroad, or from public or private research centers.
L'archive ouverte pluridisciplinaire HAL, est destinée au dépôt et à la diffusion de documents scientifiques de niveau recherche, publiés ou non, émanant des établissements d'enseignement et de recherche français ou étrangers, des laboratoires publics ou privés. 


\title{
Differentiating $\sigma$-fields for Gaussian and shifted Gaussian processes
}

\author{
Sébastien Darses*, Ivan Nourdin ${ }^{\dagger}$ and Giovanni Peccati ${ }^{\ddagger}$ \\ 31st January 2007
}

\begin{abstract}
We study the notions of differentiating and non-differentiating $\sigma$-fields in the general framework of (possibly drifted) Gaussian processes, and characterize their invariance properties under equivalent changes of probability measure. As an application, we investigate the class of stochastic derivatives associated with shifted fractional Brownian motions. We finally establish conditions for the existence of a jointly measurable version of the differentiated process, and we outline a general framework for stochastic embedded equations.
\end{abstract}

\section{Introduction}

Let $X$ be the solution of the stochastic differential equation $X_{t}=X_{0}+$ $\int_{0}^{t} \sigma\left(X_{s}\right) d B_{s}+\int_{0}^{t} b\left(X_{s}\right) d s, t \in[0, T]$, where $\sigma, b: \mathbb{R} \rightarrow \mathbb{R}$ are suitably regular functions and $B$ is a standard Brownian motion, and denote by $\mathscr{P}_{t}^{X}$ the $\sigma$-field generated by $\left\{X_{s}, s \in[0, t]\right\}$. Then, the following quantity:

$$
h^{-1} \mathrm{E}\left[f\left(X_{t+h}\right)-f\left(X_{t}\right) \mid \mathscr{P}_{t}^{X}\right]
$$

*LPMA, Université Paris 6, Boîte courrier 188, 4 Place Jussieu, 75252 Paris Cedex 05, France, sedarses@ccr.jussieu.fr

${ }^{\dagger}$ LPMA, Université Paris 6, Boîte courrier 188, 4 Place Jussieu, 75252 Paris Cedex 05, France, nourdin@ccr.jussieu.fr

${ }^{\ddagger}$ LSTA, Université Paris 6, Boîte courrier 188, 4 Place Jussieu, 75252 Paris Cedex 05, France, giovanni.peccati@gmail.com 
converges (in probability and for $h \downarrow 0$ ) for every smooth and bounded function $f$. This existence result is the key to define one of the central operators in the theory of diffusion processes: the infinitesimal generator $L$ of $X$, which is given by $L f(x)=b(x) \frac{d f}{d x}(x)+\frac{1}{2} \sigma(x)^{2} \frac{d^{2} f}{d x^{2}}(x)$ (the domain of $L$ contains all regular functions $f$ as above). Note that the limit in (11) is taken conditionally to the past of $X$ before $t$; however, due to the Markov property of $X$, one may as well replace $\mathscr{P}_{t}^{X}$ with the $\sigma$-field $\sigma\left\{X_{t}\right\}$ generated by $X_{t}$. On the other hand, under rather mild conditions on $b$ and $\sigma$, one can take $f=\operatorname{Id}$ in (1), so that the limit still exists and coincides with the natural definition of the mean velocity of $X$ at $t$ (the reader is referred to Nelson's dynamical theory of Brownian diffusions, as developed e.g. in [8], for more results in this direction - see also [1] for a recent survey).

In this paper we are concerned with the following question: is it possible to obtain the existence, and to study the nature, of limits analogous to (1), when $X$ is neither a Markov process nor a semimartingale? We will mainly focus on the case where $X$ is a (possibly shifted) Gaussian random process and $f=$ Id (the case of a non-linear and smooth $f$ will be investigated elsewhere). The subtleties of the problem are better appreciated through an example. Consider for instance a fractional Brownian motion (fBm) $B$ of Hurst index $H \in(1 / 2,1)$, and recall that $B$ is neither Markovian nor a semimartingale (see e.g. [9]). Then, the quantity $h^{-1} \mathrm{E}\left[B_{t+h}-B_{t} \mid B_{t}\right]$ converges in $L^{2}(\Omega)$ (as $h \downarrow 0$ ), while the quantity $h^{-1} \mathrm{E}\left[B_{t+h}-B_{t} \mid \mathscr{P}_{t}^{B}\right]$ does not admit a limit in probability. More to the point, similar properties can be shown to hold also for suitably regular solutions of stochastic differential equations driven by $B$ (see [3] for precise statements and proofs).

To address the problem evoked above, we shall mainly use the notion of differentiating $\sigma$-field introduced in [3]: if $Z$ is a process defined on a probability space $(\Omega, \mathscr{F}, \mathbf{P})$, we say that a $\sigma$-field $\mathscr{G} \subset \mathscr{F}$ is differentiating for $Z$ at $t$ if

$$
h^{-1} \mathrm{E}\left[Z_{t+h}-Z_{t} \mid \mathscr{G}\right]
$$

converges in some topology, when $h$ tends to 0 . When it exists, the limit of (2) is noted $D^{\mathscr{G}} Z_{t}$, and it is called the stochastic derivative of $Z$ at $t$ with respect to $\mathscr{G}$. Note that if a sub- $\sigma$-field $\mathscr{G}$ of $\mathscr{F}$ is not differentiating, one can implement two "strategies" to make (2) converge: either one replaces $\mathscr{G}$ with a differentiating sub- $\sigma$-field $\mathscr{H}$, or one replaces $h^{-1}$ with $h^{-\alpha}$ with $0<\alpha<1$. In particular, the second strategy pays dividends when a non-differentiating $\sigma$-field $\mathscr{G}$ is too poor, in the sense that $\mathscr{G}$ does not contain sufficiently good differentiating $\sigma$-fields. We will see that this is exactly the case for a $\mathrm{fBm} B$ with index $H<1 / 2$, when $\mathscr{G}$ is generated by $B_{s}$ for some $s>0$.

The aim of this paper is to give a precise characterization of the classes of differentiating and non differentiating $\sigma$-fields for Gaussian and shifted Gaussian processes. 
We will systematically investigate their mutual relations, and pay special attention to their invariance properties under equivalent changes of probability measure.

The paper is organized as follows. In Sections 2 and 3 we introduce several notions related to the concept of differentiating $\sigma$-field, and give a characterization of differentiating and non differentiating $\sigma$-fields in a Gaussian framework. In Section 4 we prove some invariance properties of differentiating $\sigma$-fields under equivalent changes of probability measure. Notably, we will be able to write an explicit relation between the stochastic derivatives associated with different probabilities. We will illustrate our results by considering the example of shifted fractional Brownian motions, and we shall pinpoint different behaviors when the Hurst index is, respectively, in $(0,1 / 2)$ and in $(1 / 2,1)$. In Section 5 we establish fairly general conditions, ensuring the existence of a jointly measurable version of the differentiated process induced by a collection of differentiating $\sigma$-fields. Finally, in Section 6 we outline a general framework for embedded ordinary stochastic differential equations (as defined in [2]) and we analyze a simple example.

\section{Preliminaries on stochastic derivatives}

Let $\left(Z_{t}\right)_{t \in[0, T]}$ be a stochastic process defined on a probability space $(\Omega, \mathscr{F}, \mathbf{P})$. In the sequel, we will always assume that $Z_{t} \in \mathrm{L}^{2}(\Omega, \mathscr{F}, \mathbf{P})$ for every $t \in[0, T]$. It will also be implicit that each $\sigma$-field we consider is a sub- $\sigma$-field of $\mathscr{F}$; analogously, given a $\sigma$-field $\mathscr{H}$, the notation $\mathscr{G} \subset \mathscr{H}$ will mean that $\mathscr{G}$ is a sub- $\sigma$-field of $\mathscr{H}$. For every $t \in(0, T)$ and every $h \neq 0$ such that $t+h \in(0, T)$, we set

$$
\Delta_{h} Z_{t}=\frac{Z_{t+h}-Z_{t}}{h} .
$$

For the rest of the paper, we will use the letter $\tau$ as a generic symbol to indicate a topology on the class of real-valued and $\mathscr{F}$-measurable random variables. For instance, $\tau$ can be the topology induced either by the a.s. convergence, or by the $\mathrm{L}^{p}$ convergence $(p \geqslant 1)$, or by the convergence in probability, or by both a.s. and $L^{p}$ convergences, in which cases we shall write, respectively,

$$
\tau=\text { a.s. }, \quad \tau=\mathrm{L}^{p}, \quad \tau=\text { proba }, \quad \tau=\mathrm{L}^{p} \star \text { a.s.. }
$$

Note that, when no further specification is provided, any convergence is tacitly defined with respect to the reference probability measure $\mathbf{P}$.

Definition 1 Fix $t \in(0, T)$ and let $\mathscr{G} \subset \mathscr{F}$. We say that $\mathscr{G} \tau$-differentiates $Z$ at $t$ if

$$
\mathrm{E}\left[\Delta_{h} Z_{t} \mid \mathscr{G}\right] \text { converges w.r.t. } \tau \text { when } h \rightarrow 0 \text {. }
$$


In this case, we define the so-called $\tau$-stochastic derivative of $Z$ w.r.t. $\mathscr{G}$ at $t$ by

$$
D_{\tau}^{\mathscr{G}} Z_{t}=\tau-\lim _{h \rightarrow 0} \mathrm{E}\left[\Delta_{h} Z_{t} \mid \mathscr{G}\right] .
$$

If the limit in (3) does not exist, we say that $\mathscr{G}$ does not $\tau$-differentiate $Z$ at $t$. If there is no risk of ambiguity on the topology $\tau$, we will write $D^{\mathscr{G}} Z_{t}$ instead of $D_{\tau}^{\mathscr{G}} Z_{t}$ to simplify the notation.

Remark. When $\tau=$ a.s. (i.e., when $\tau$ is the topology induced by a.s. convergence), equation (3) must be understood in the following sense (note that, in (3), $t$ acts as a fixed parameter): there exists a jointly measurable application $(\omega, h) \mapsto q(\omega, h)$, from $\Omega \times(-\varepsilon, \varepsilon)$ to $\mathbb{R}$, such that (i) $q(\cdot, h)$ is a version of $\mathrm{E}\left[\Delta_{h} Z_{t} \mid \mathscr{G}\right]$ for every fixed $h$, and (ii) there exists a set $\Omega^{\prime} \subset \Omega$, of $\mathbf{P}$-probability one, such that $q(\omega, h)$ converges, as $h \rightarrow 0$, for every $\omega \in \Omega^{\prime}$. An analogous remark applies to the case $\tau=\mathrm{L}^{p} \star$ a.s. $(p \geqslant 1)$.

The set of all $\sigma$-fields that $\tau$-differentiate $Z$ at time $t$ is denoted by $\mathscr{M}_{Z}^{(t), \tau}$. Intuitively, one can say that the more $\mathscr{M}_{Z}^{(t), \tau}$ is large, the more $Z$ is regular at time $t$. For instance, one has clearly that $\{\emptyset, \Omega\} \in \mathscr{M}_{Z}^{(t), \tau}$ if, and only if, the application $s \mapsto \mathrm{E}\left(Z_{s}\right)$ is differentiable at time $t$. On the other hand, $\mathscr{F} \in \mathscr{M}_{Z}^{(t), \tau}$ if, and only if, the random function $s \mapsto Z_{s}$ is $\tau$-differentiable at time $t$.

Before introducing some further definitions, we shall illustrate the above notions by a simple example involving the $\mathrm{L}^{2} \star$ a.s.-topology. Assume that $Z=\left(Z_{t}\right)_{t \in[0, T]}$ is a Gaussian process such that $\operatorname{Var}\left(Z_{t}\right) \neq 0$ for every $t \in(0, T]$. Fix $t \in(0, T)$ and take $\mathscr{G}$ to be the present of $Z$ at a fixed time $s \in(0, T]$, that is, $\mathscr{G}=\sigma\left\{Z_{s}\right\}$ is the $\sigma$-field generated by $Z_{s}$. Since one has, by linear regression,

$$
\mathrm{E}\left[\Delta_{h} Z_{t} \mid \mathscr{G}\right]=\frac{\operatorname{Cov}\left(\Delta_{h} Z_{t}, Z_{s}\right)}{\operatorname{Var}\left(Z_{s}\right)} Z_{s}
$$

we immediately deduce that $\mathscr{G}$ differentiates $Z$ at $t$ if, and only if,

$$
\left.\frac{d}{d u} \operatorname{Cov}\left(Z_{u}, Z_{s}\right)\right|_{u=t}
$$

exists (see also Lemma 1). Now, let $\mathscr{H}$ be a $\sigma$-field such that $\mathscr{H} \subset \mathscr{G}$. Owing to the projection principle, one can write:

$$
\mathrm{E}\left[\Delta_{h} Z_{t} \mid \mathscr{H}\right]=\frac{\operatorname{Cov}\left(\Delta_{h} Z_{t}, Z_{s}\right)}{\operatorname{Var}\left(Z_{s}\right)} \mathrm{E}\left[Z_{s} \mid \mathscr{H}\right],
$$

and we conclude that 
(A) If $\mathscr{G}$ differentiates $Z$ at $t$, then it is also the case for any $\mathscr{H} \subset \mathscr{G}$.

(B) If $\mathscr{G}$ does not differentiates $Z$ at $t$, then any $\mathscr{H} \subset \mathscr{G}$ either does not differentiates $Z$ at $t$, or (when $\mathrm{E}\left[Z_{s} \mid \mathscr{H}\right]=0$ ) differentiates $Z$ at $t$ with $D^{\mathscr{H}} Z_{t}=0$

The phenomenon appearing in (A) is quite natural, not only in a Gaussian setting, and it is due to the well-known properties of conditional expectations: see Proposition 11 below. On the other hand, (B) seems strongly linked to the Gaussian assumptions we made on $Z$. We shall use fine arguments to generalize (B) to a non-Gaussian framework, see Sections 3 and 1 below.

This example naturally leads to the subsequent definitions.

Definition 2 Fix $t \in(0, T)$ and let $\mathscr{G} \subset \mathscr{F}$. If $\mathscr{G} \tau$-differentiates $Z$ at $t$ and if we have $D_{\tau}^{\mathscr{G}} Z_{t}=c$ a.s. for a certain real $c \in \mathbb{R}$, we say that $\mathscr{G} \tau$-degenerates $Z$ at $t$. We say that a random variable $Y \tau$-degenerates $Z$ at $t$ if the $\sigma$-field $\sigma\{Y\}$ generated by $Y \tau$-degenerates $Z$ at $t$.

If $D_{\tau}^{\mathscr{G}} Z_{t} \in \mathrm{L}^{2}$ (for instance when we choose $\tau=\mathrm{L}^{2}$, or $\tau=\mathrm{L}^{2} \star$ a.s., etc.), the condition on $D_{\tau}^{\mathscr{G}} Z_{t}$ in the previous definition is obviously equivalent to $\operatorname{Var}\left(D_{\tau}^{\mathscr{G}} Z_{t}\right)=0$. For instance, if $Z$ is a process such that $s \rightarrow \mathrm{E}\left(Z_{s}\right)$ is differentiable in $t \in(0, T)$ then $\{\emptyset, \Omega\}$ degenerates $Z$ at $t$.

Definition 3 Let $t \in(0, T)$ and $\mathscr{G} \subset \mathscr{F}$. We say that $\mathscr{G}$ really does not $\tau$ differentiate $Z$ at $t$ if $\mathscr{G}$ does not $\tau$-differentiate $Z$ at $t$ and if any $\mathscr{H} \subset \mathscr{G}$ either $\tau$-degenerates $Z$ at $t$, or does not $\tau$-differentiate $Z$ at $t$.

Consider e.g. the phenomenon described at point (B) above: the $\sigma$-field $\mathscr{G} \triangleq \sigma\left\{Z_{s}\right\}$ really does not differentiate the Gaussian process $Z$ at $t$ whenever $\left.\frac{d}{d u} \operatorname{Cov}\left(Z_{u}, Z_{s}\right)\right|_{u=t}$ does not exist, since every $\mathscr{H} \subset \mathscr{G}$ either does not differentiate or degenerates $Z$ at $t$. It is for instance the case when $Z=B$ is a fractional Brownian motion with Hurst index $H<1 / 2$ and $s=t$, see Corollary 2 . Another interesting example is given by the process $Z_{t}=f_{1}(t) N_{1}+f_{2}(t) N_{2}$, where $f_{1}, f_{2}:[0, T] \rightarrow \mathbb{R}$ are two deterministic functions and $N_{1}, N_{2}$ are two centered and independent random variables. Assume that $f_{1}$ is differentiable at $t \in(0, T)$ but that $f_{2}$ is not. This yields that $\mathscr{G} \triangleq \sigma\left\{N_{1}, N_{2}\right\}$ does not differentiates $Z$ at $t$. Moreover, one can easily show that $\mathscr{H} \triangleq \sigma\left\{N_{1}\right\} \subset \mathscr{G}$ differentiates $Z$ at $t$ with $D^{\mathscr{H}} Z_{t}=f_{1}^{\prime}(t) N_{1}$, which is not constant in general. Then, although $\mathscr{G}$ does not differentiate $Z$ at $t$, it does not meet the requirements of Definition 0 . 


\section{Stochastic derivatives and Gaussian processes}

In this section we mainly focus on Gaussian processes, and we shall systematically work with the $\mathrm{L}^{2}$ - or the $\mathrm{L}^{2} \star$ a.s.-topology, which are quite natural in this framework. In the sequel we will also omit the symbol $\tau$ in (4), as we will always indicate the topology we are working with.

Our aim is to establish several relationships between differentiating and (really) non differentiating $\sigma$-fields under Gaussian-type assumptions. However, our first result pinpoints a general simple fact, which also holds in a non-Gaussian framework, that is: any sub- $\sigma$-field of a differentiating $\sigma$-field is also differentiating.

Proposition 1 Let $Z$ be a stochastic process (not necessarily Gaussian) such that $Z_{t} \in \mathrm{L}^{2}(\Omega, \mathscr{F}, \mathbf{P})$ for every $t \in(0, T)$. Let $t \in(0, T)$ be fixed, and let $\mathscr{G} \subset \mathscr{F}$. If $\mathscr{G}$ $\mathrm{L}^{2}$-differentiates $Z$ at $t$, then any $\mathscr{H} \subset \mathscr{G}$ also $\mathrm{L}^{2}$-differentiates $Z$ at $t$. Moreover, we have

$$
D^{\mathscr{H}} Z_{t}=\mathrm{E}\left[D^{\mathscr{G}} Z_{t} \mid \mathscr{H}\right] .
$$

Proof: We can write, by the projection principle and Jensen inequality:

$$
\begin{aligned}
\mathrm{E}\left[\left(\mathrm{E}\left(\Delta_{h} Z_{t} \mid \mathscr{H}\right)-\mathrm{E}\left(D^{\mathscr{G}} Z_{t} \mid \mathscr{H}\right)\right)^{2}\right] & =\mathrm{E}\left[\mathrm{E}\left[\mathrm{E}\left(\Delta_{h} Z_{t}-D^{\mathscr{G}} Z_{t} \mid \mathscr{G}\right) \mid \mathscr{H}\right]^{2}\right] \\
& \leq \mathrm{E}\left[\left(\mathrm{E}\left(\Delta_{h} Z_{t} \mid \mathscr{G}\right)-D^{\mathscr{G}} Z_{t}\right)^{2}\right] .
\end{aligned}
$$

So, the $\mathrm{L}^{2}$-convergence of $\mathrm{E}\left(\Delta_{h} Z_{t} \mid \mathscr{H}\right)$ to $\mathrm{E}\left(D^{\mathscr{G}} Z_{t} \mid \mathscr{H}\right)$ as $h \rightarrow 0$ is obvious.

On the other hand, a non differentiating $\sigma$-field may contain a differentiating $\sigma$-field (for instance, when the non differentiating $\sigma$-field is generated both by differentiating and non differentiating random variables).

We now provide a characterization of the really non-differentiating $\sigma$-fields that are generated by some subspace of the first Wiener chaos associated with a centered Gaussian process $Z$, noted $\mathcal{H}_{1}(Z)$. We recall that $\mathcal{H}_{1}(Z)$ is the $\mathrm{L}^{2}$-closed linear vector space generated by random variables of the type $Z_{t}, t \in[0, T]$.

Theorem 1 Let $I=\{1,2, \ldots, N\}$, with $N \in \mathbb{N}^{*} \cup\{+\infty\}$ and let $Z=\left(Z_{t}\right)_{t \in[0, T]}$ be a centered Gaussian process. Fix $t \in(0, T)$, and consider a subset $\left\{Y_{i}\right\}_{i \in I}$ of $\mathcal{H}_{1}(Z)$ such that, for any $n \in I$, the covariance matrix $M_{n}$ of $\left\{Y_{i}\right\}_{1 \leq i \leq n}$ is invertible. Finally, note $\mathscr{Y}=\sigma\left\{Y_{i}, i \in I\right\}$. Then:

1. If $\mathscr{Y} \mathrm{L}^{2}$-differentiates $Z$ at $t$, then, for any $i \in I, Y_{i} \mathrm{~L}^{2}$-differentiates $Z$ at $t$. If $N<+\infty$, the converse also holds. 
2. Suppose $N<+\infty$. Then $\mathscr{Y}$ really does not $\mathrm{L}^{2} \star$ a.s.-differentiate $Z$ at $t$ if, and only if, any finite linear combination of the $Y_{i}$ 's either $\mathrm{L}^{2} \star$ a.s.-degenerates or does not $\mathrm{L}^{2} \star$ a.s.-differentiate $Z$ at $t$.

3. Suppose that $N=+\infty$ and that the sequence $\left\{Y_{i}\right\}_{i \in I}$ is i.i.d.. Write moreover $\mathbf{R}(\mathscr{Y})$ to indicate the class of all the sub- $\sigma$-fields of $\mathscr{Y}$ that are generated by rectangles of the type $A_{1} \times \cdots \times A_{d}$, with $A_{i} \in \sigma\left\{Y_{i}\right\}$, and $d \geqslant 1$. Then, the previous characterization holds in a weak sense: if $\mathscr{Y}$ really does not $\mathrm{L}^{2} \star$ a.s.differentiate $Z$ at $t$, then every finite linear combination of the $Y_{i}$ 's either $\mathrm{L}^{2} \star$ a.s.-degenerates or does not $\mathrm{L}^{2} \star$ a.s.-differentiate $Z$ at $t$; on the other hand, if every finite linear combination of the $Y_{i}$ 's either $\mathrm{L}^{2} \star$ a.s. -degenerates or does not $\mathrm{L}^{2} \star$ a.s.-differentiate $Z$ at $t$, then any $\mathscr{G} \in \mathbf{R}(\mathscr{Y})$ either $\mathrm{L}^{2} \star$ a.s.-degenerates or does not $\mathrm{L}^{2} \star$ a.s.-differentiate $Z$ at $t$.

The class $\mathbf{R}(\mathscr{Y})$ contains for instance the $\sigma$-fields of the type

$$
\mathscr{G}=\sigma\left\{f_{1}\left(Y_{1}\right), \ldots, f_{d}\left(Y_{d}\right)\right\}
$$

where $d \geqslant 1$. When $N=1$, the second point of Theorem [ can be reformulated as follows (see also the examples discussed in Section 2 above).

Corollary 1 Let $Z=\left(Z_{t}\right)_{t \in[0, T]}$ be a centered Gaussian process and let $\mathcal{H}_{1}(Z)$ be its first Wiener chaos. Fix $t \in(0, T)$, as well as $Y \in \mathcal{H}_{1}(Z)$, and set $\mathscr{Y}=\sigma\{Y\}$. Then, $\mathscr{Y}$ does not $\mathrm{L}^{2}$-differentiate $Z$ at $t$ (resp. $\mathrm{L}^{2} \star$ a.s.) if, and only if, $\mathscr{Y}$ really does not $\mathrm{L}^{2}$-differentiate $Z$ at $t$ (resp. $\mathrm{L}^{2} \star$ a.s.).

In particular, when $Z=B$ is a fractional Brownian motion with Hurst index $H \in$ $(0,1 / 2) \cup(1 / 2,1), t$ is a fixed time in $(0, T)$ and $\mathscr{Y}=\sigma\left\{B_{t}\right\}$ is the present of $B$ at time $t$, we observe two distinct behaviors, according to the different values of $H$ :

(a) If $H>1 / 2$, then $\mathscr{Y} \mathrm{L}^{2} \star$ a.s.-differentiates $B$ at $t$ and it is also the case for any $\mathscr{Y}_{0} \subset \mathscr{Y}$.

(b) If $H<1 / 2$, then $\mathscr{Y}$ really does not $\mathrm{L}^{2} \star$ a.s.-differentiate $B$ at $t$.

Indeed, (a) and (b) are direct consequences of Proposition 1, Corollary 1 and the equality

$$
\mathrm{E}\left[\Delta_{h} B_{t} \mid B_{t}\right]=\frac{(t+h)^{2 H}-t^{2 H}-|h|^{2 H}}{2 t^{2 H} h} B_{t}
$$

which is immediately verified by a Gaussian linear regression.

Note that [3, Theorem 22] generalizes (a) to the case of fractional diffusions. In the subsequent sections, we will propose a generalization of (a) and (b) to the case of shifted fractional Brownian motions - see Proposition 2 .

In order to prove Theorem 1, we state an easy but quite useful lemma: 
Lemma 1 Let $Z=\left(Z_{t}\right)_{t \in[0, T]}$ be a centered Gaussian process, and let $\mathcal{H}_{1}(Z)$ be its first Wiener chaos. Fix $Y \in \mathcal{H}_{1}(Z)$ and $t \in(0, T)$. Then, the following assertions are equivalent:

(a) $Y$ a.s.-differentiates $Z$ at $t$.

(b) $Y \mathrm{~L}^{2}$-differentiates $Z$ at $t$.

(c) $\left.\frac{d}{d s} \operatorname{Cov}\left(Z_{s}, Y\right)\right|_{s=t}$ exists and is finite.

If either (a), (b) or (c) are verified and $P(Y=0)<1$, one has moreover that

$$
D^{Y} Z_{t}=\left.\frac{Y}{\operatorname{Var}(Y)} \cdot \frac{d}{d s} \operatorname{Cov}\left(Z_{s}, Y\right)\right|_{s=t} .
$$

In particular, for every $s, t \in(0, T)$, we have: $Z_{s} \mathrm{~L}^{2} \star$ a.s.-differentiates $Z$ at $t$ if, and only if, $u \mapsto \operatorname{Cov}\left(Z_{s}, Z_{u}\right)$ is differentiable at $u=t$.

On the other hand, suppose that $Y \in \mathcal{H}_{1}(Z)$ is such that: (i) $P(Y=0)<1$, and (ii) $Y$ does not $\mathrm{L}^{2} \star$ a.s. -differentiate $Z$ at $t \in(0, T)$. Then, for every $\mathscr{H} \subset \sigma\{Y\}$, either $\mathscr{H}$ does not $\mathrm{L}^{2} \star$ a.s.-differentiate $Z$ at $t$, or $\mathscr{H}$ is such that $\mathrm{E}[Y \mid \mathscr{H}]=0$ and $D^{\mathscr{H}} Z_{t}=0$.

Proof: If $Y \in \mathcal{H}_{1}(Z) \backslash\{0\}$, we have

$$
\mathrm{E}\left[\Delta_{h} Z_{t} \mid Y\right]=\frac{\operatorname{Cov}\left(\Delta_{h} Z_{t}, Y\right)}{\operatorname{Var}(Y)} Y .
$$

The conclusions follow.

We now turn to the proof of Theorem [1:

Proof: Since $M_{n}$ is an invertible matrix for any $n \in I$, the Gram-Schmidt orthonormalization procedure can be applied to $\left\{Y_{i}\right\}_{i \in I}$. For this reason we may assume, for the rest of the proof and without loss of generality, that the family $\left\{Y_{i}\right\}_{i \in I}$ is composed of i.i.d. random variables with common law $\mathscr{N}(0,1)$.

1. The first implication is an immediate consequence of Proposition 1. Assume now that $N<+\infty$ and that any $Y_{i}, i=1, \ldots, N, \mathrm{~L}^{2}$-differentiates $Z$ at $t$. By Lemma 1, we have in particular that

$$
\left.\frac{d}{d s} \operatorname{Cov}\left(Z_{s}, Y_{i}\right)\right|_{s=t}
$$


exists for any $i=1, \ldots, N$. Since

$$
\mathrm{E}\left[\Delta_{h} Z_{t} \mid \mathscr{Y}\right]=\sum_{i=1}^{N} \operatorname{Cov}\left(\Delta_{h} Z_{t}, Y_{i}\right) Y_{i}
$$

we deduce that $\mathscr{Y} \mathrm{L}^{2}$-differentiates $Z$ at $t$.

2. By definition, if $\mathscr{Y}$ really does not $L^{2} \star$ a.s.-differentiate $Z$ at $t$, then any finite linear combination of the $Y_{i}$ 's either $\mathrm{L}^{2} \star$ a.s.-degenerates, or does not $\mathrm{L}^{2} \star$ a.s.differentiate $Z$ at $t$.

Conversely, assume that any finite linear combination of the $Y_{i}$ 's either $\mathrm{L}^{2} \star$ a.s.degenerates or does not $\mathrm{L}^{2} \star$ a.s.-differentiate $Z$ at $t$. Let $\mathscr{G} \subset \mathscr{Y}$. By the projection principle, we can write:

$$
\mathrm{E}\left[\Delta_{h} Z_{t} \mid \mathscr{G}\right]=\sum_{i \in I} \operatorname{Cov}\left(\Delta_{h} Z_{t}, Y_{i}\right) \mathrm{E}\left[Y_{i} \mid \mathscr{G}\right]
$$

Let us assume that $\mathscr{G} \mathrm{L}^{2} \star$ a.s.-differentiates $Z$ at $t$. By (8) this implies in particular that, for almost all fixed $\omega_{0} \in \Omega$,

$$
\mathrm{E}\left[\Delta_{h} Z_{t} \mid \mathscr{G}\right]\left(\omega_{0}\right)=\operatorname{Cov}\left(\Delta_{h} Z_{t}, \sum_{i=1}^{N} a_{i}\left(\omega_{0}\right) Y_{i}\right),
$$

converges as $h \rightarrow 0$, where $a_{i}\left(\omega_{0}\right)=\mathrm{E}\left[Y_{i} \mid \mathscr{G}\right]\left(\omega_{0}\right)$. Due to Lemma 1, we deduce that $X^{\left(\omega_{0}\right)} \triangleq \sum_{i=1}^{N} a_{i}\left(\omega_{0}\right) Y_{i} \mathrm{~L}^{2} \star$ a.s.-differentiates $Z$ at $t$ for almost all $\omega_{0} \in \Omega$. By hypothesis, we deduce that $X^{\left(\omega_{0}\right)} \mathrm{L}^{2} \star$ a.s.-degenerates $Z$ at $t$ for almost all $\omega_{0} \in \Omega$. But, by Lemma 1, the stochastic derivative $D^{X^{\left(\omega_{0}\right)}} Z_{t}$ necessarily writes $c\left(\omega_{0}\right) X^{\left(\omega_{0}\right)}$ with $c\left(\omega_{0}\right) \in \mathbb{R}$. Since $X^{\left(\omega_{0}\right)}$ is centered and $\operatorname{Var}\left(D^{X^{\left(\omega_{0}\right)}} Z_{t}\right)=0$, we deduce that $D^{X^{\left(\omega_{0}\right)}} Z_{t}=0$. Thus

$$
\lim _{h \rightarrow 0} \operatorname{Cov}\left(\Delta_{h} Z_{t}, X^{\left(\omega_{0}\right)}\right)=\lim _{h \rightarrow 0} \mathrm{E}\left[\Delta_{h} Z_{t} \mid \mathscr{G}\right]\left(\omega_{0}\right)=0
$$

for almost all $\omega_{0} \in \Omega$. Thus $\mathscr{G}$ a.s.-degenerates $Z$ at $t$. Since $\mathscr{G}$ also $\mathrm{L}^{2}$ differentiates $Z$ at $t$, we conclude that $\mathscr{G} \mathrm{L}^{2} \star$ a.s.-degenerates $Z$ at $t$. The proof that $\mathscr{Y}$ really does not $\mathrm{L}^{2} \star$ a.s.-differentiate $Z$ at $t$ is complete.

3. Again by definition, if $\mathscr{Y}$ really does not $\mathrm{L}^{2} \star$ a.s.-differentiate $Z$ at $t$, then any finite linear combination of the $Y_{i}$ 's either $\mathrm{L}^{2} \star$ a.s.-degenerates, or does not $\mathrm{L}^{2} \star$ a.s.-differentiate $Z$ at $t$. We shall now assume that every finite linear combination of the $Y_{i}$ 's either $\mathrm{L}^{2} \star$ a.s.-degenerates or does not $\mathrm{L}^{2} \star$ a.s.-differentiate 
$Z$ at $t$. Let $\left(J_{m}\right)_{m \in \mathbb{N}}$ be the increasing sequence given by $J_{m}=\{1, \ldots, m\}$, so that $\cup_{m \in \mathbb{N}} J_{m}=I=\mathbb{N}$.

Suppose that $\mathscr{G} \in \mathbf{R}(\mathscr{Y})$ and that $\mathscr{G} \mathrm{L}^{2} \star$ a.s.-differentiates $Z$ at $t$. By Proposition 1, $\mathscr{G}^{i} \triangleq \mathscr{G} \cap \sigma\left\{Y_{i}\right\} \mathrm{L}^{2}$-differentiates $Z$ at $t$, for any $i \in \mathbb{N}$. But

$$
\mathrm{E}\left[\Delta_{h} Z_{t} \mid \mathscr{G}^{i}\right]=\operatorname{Cov}\left(\Delta_{h} Z_{t}, Y_{i}\right) E\left[Y_{i} \mid \mathscr{G}^{i}\right] .
$$

So, for any $i \in \mathbb{N}$ :

$$
\text { either } \lim _{h \rightarrow 0} \operatorname{Cov}\left(\Delta_{h} Z_{t}, Y_{i}\right) \text { exists, or } \mathrm{E}\left[Y_{i} \mid \mathscr{G}^{i}\right]=0 \text {. }
$$

Set $\mathscr{G}_{m} \triangleq \mathscr{G} \cap \sigma\left(Y_{j}, j \in J_{m}\right)$, and observe that, if $\mathscr{G} \in \mathbf{R}(\mathscr{Y})$, then

$$
\mathrm{E}\left[Y_{i} \mid \mathscr{G}^{i}\right]=\mathrm{E}\left[Y_{i} \mid \mathscr{G}_{m}\right]
$$

for every $i=1, \ldots, m$. We have

$$
\mathrm{E}\left[\Delta_{h} Z_{t} \mid \mathscr{G}_{m}\right]=\sum_{i \in J_{m}} \operatorname{Cov}\left(\Delta_{h} Z_{t}, Y_{i}\right) \mathrm{E}\left[Y_{i} \mid \mathscr{G}^{i}\right] .
$$

By (9), and since $J_{m}$ is finite, we deduce that $\mathscr{G}_{m} \mathrm{~L}^{2} \star$ a.s.-differentiates $Z$ at $t$. By the same proof as in step (a) for $\mathscr{G}_{m}$ instead of $\mathscr{G}$ and using (10) instead of (8), we deduce that

$$
X_{m}^{(t)} \triangleq D^{\mathscr{G}_{m}} Z_{t}=0
$$

But, from Proposition 1, we have:

$$
D^{\mathscr{G}_{m}} Z_{t}=\mathrm{E}\left[D^{\mathscr{G}} Z_{t} \mid \mathscr{G}_{m}\right], \quad m \geqslant 1 .
$$

Thus $\left\{X_{m}^{(t)}, m \in \mathbb{N}\right\}$ is a (discrete) square integrable martingale w.r.t. the filtration $\left\{\mathscr{G}_{m}, m \in \mathbb{N}\right\}$. So we conclude that

$$
D^{\mathscr{G}} Z_{t}=\lim _{m \rightarrow \infty} X_{m}^{(t)}=0 \quad \text { a.s. }
$$

In other words, $\mathscr{G} \mathrm{L}^{2} \star$ a.s.-degenerates $Z$ at $t$. Therefore, $\mathscr{Y}$ really does not $\mathrm{L}^{2} \star$ a.s.-differentiate $Z$ at $t$.

Counterexample. In what follows we show that, if $N=+\infty$, the converse of the first point in the statement of Theorem 1 does not hold in general. Indeed, let $\left\{\xi_{i}: i \geqslant 1\right\}$ be an infinite sequence of i.i.d. centered standard Gaussian random variables. Let $\left\{f_{i}: i \geqslant 1\right\}$ be a collection of deterministic functions belonging to $L^{2}([0,1], d t)$, such that the following hold: 
- for every $i \geqslant 1, f_{i}(t)$ is differentiable in $t$ for every $t \in[0,1]$;

- there exists $A \in(0,+\infty)$ such that, for every $t \in[0,1], \sum_{i=1}^{+\infty} f_{i}(t)^{2}<A$.

Then, we may apply the Itô-Nisio theorem (see [6]) to deduce that there exists a Gaussian process $\left\{Z_{t}: t \in[0,1]\right\}$ such that, a.s.- $\mathbb{P}$,

$$
\lim _{N \rightarrow+\infty} \sup _{t \in[0,1]}\left|Z_{t}-\sum_{i=1}^{N} \xi_{i} f_{i}(t)\right|=0 .
$$

Now suppose that the paths of $Z$ are a.s. not-differentiable for every $t$. Then, by setting $\mathscr{Y}=\sigma\left(\xi_{i}, i \geqslant 1\right)$, we obtain that $\mathscr{Y}$ does not $\mathrm{L}^{2} \star$ a.s. differentiate $Z$ at every $t$, although, for every $i \geqslant 1$ and every $t \in[0,1], \xi_{i} \mathrm{~L}^{2} \star$ a.s. differentiates $Z$ at $t$. As an example, one can consider the case

$$
f_{i}(t)=\int_{0}^{t} e_{i}(x) d x, \quad i \geqslant 1,
$$

where $\left\{e_{i}: i \geqslant 1\right\}$ is any orthonormal basis of $L^{2}([0,1], d x)$, so that the limit process $Z$ is a standard Brownian motion. See also Kadota [7] for several related results, concerning the differentiability of stochastic processes admitting a Karhunen-Loève type expansion.

\section{Invariance properties of differentiating $\sigma$-fields and stochastic deriva- tives under equivalent changes of probability}

Let $Z$ be a Gaussian process, and let $\mathscr{G} \subset \mathscr{F}$ be differentiating for $Z$. In this section we establish conditions on $Z$ and $\mathscr{G}$, ensuring that $\mathscr{G}$ is still differentiating for $Z$ after an equivalent change of probability measure. As anticipated, this result will be used to study the class of differentiating $\sigma$-fields associated with drifted Gaussian processes. Roughly speaking, we will show that - under adequate conditions - one can study the stochastic derivatives of a drifted Gaussian process by first eliminating the drift through a Girsanov-type transformation. We concentrate on $\sigma$-fields generated by a single random variable. To achieve our goals we will use several techniques from Malliavin calculus, as for instance those developed by H. Föllmer (see [5, Sec. 4]) in order to compute the backward drift of a non-Markovian Brownian diffusion.

Let $Z=\left(Z_{t}\right)_{t \in[0, T]}$ be a square integrable stochastic process defined on a probability space $(\Omega, \mathscr{F}, \mathbf{P})$. We assume that, under an equivalent probability $\mathbf{Q} \sim \mathbf{P}$, $Z$ is a centered Gaussian process (so that, in particular, $Z_{t} \in \mathrm{L}^{2}(\mathbf{P}) \cap \mathrm{L}^{2}(\mathbf{Q})$ for every t). Let $\mathcal{H}_{1}(Z, \mathbf{Q})=\{Z(h), h \in \mathfrak{H}\}$ be the first Wiener chaos associated with $Z$ 
under $\mathbf{Q}$ (this means that the closure is in $\mathrm{L}^{2}(\mathbf{Q})$ ), canonically represented as an isonormal Gaussian process with respect to a separable Hilbert space $\left(\mathfrak{H},\langle\cdot, \cdot\rangle_{\mathfrak{H}}\right)$. In particular: (i) the space $\mathfrak{H}$ contains the set $\mathcal{E}$ of step functions on $[0, T]$, (ii) the covariance function of $Z$ under $\mathbf{Q}$ is given by $\rho_{\mathbf{Q}}(s, t)=\left\langle\mathbf{1}_{[0, s]}, \mathbf{1}_{[0, t]}\right\rangle_{\mathfrak{H}}$, and (iii) the scalar product $\langle\cdot, \cdot\rangle_{\mathfrak{H}}$ verifies the general relation:

$$
\forall h, h^{\prime} \in \mathfrak{H}, \quad\left\langle h, h^{\prime}\right\rangle_{\mathfrak{H}}=\mathrm{E}^{\mathbf{Q}}\left[Z(h) Z\left(h^{\prime}\right)\right]
$$

(note that, given $Z$, the properties (i)-(iii) completely characterize the pair $\left(\mathfrak{H},\langle\cdot, \cdot\rangle_{\mathfrak{H}}\right)$ ). We denote by $D$ the Malliavin derivative associated with the process $Z$ under $\mathbf{Q}$ (the reader is referred to [9] for more details about these notions). The following result is an extension of Theorem 22 in [3] to a general Gaussian setting. Note that, in the following statements, we will exclusively refer to the $\mathrm{L}^{2}$ topology.

Theorem 2 Fix $t \in(0, T)$ and select $g \in \mathfrak{H}$ such that $\left\langle\mathbf{1}_{[0, t]}, g\right\rangle_{\mathfrak{H}} \neq 0$. We write $\eta$ to indicate the Radon-Nikodym derivative of $\mathbf{Q}$ with respect to $\mathbf{P}$ (that is, $d \mathbf{Q}=\eta d \mathbf{P}$ ), and we assume that $\eta$ has the form $\eta=\exp (-\zeta)$, for some random variable $\zeta$ for which $D \zeta$ exists. Suppose that

$$
\mu_{t} \triangleq \lim _{h \rightarrow 0} h^{-1}\left\langle\mathbf{1}_{[t, t+h]}, D \zeta\right\rangle_{\mathfrak{H}} \quad \text { exists in the } \mathrm{L}^{2} \text { topology. }
$$

Then, $Z(g) \mathrm{L}^{2}$-differentiates $Z$ at $t$ under $\mathbf{P}$ if, and only if, $Z(g) \mathrm{L}^{2}$-differentiates $Z$ at $t$ under $\mathbf{Q}$, that is, if, and only if,

$$
\left.\frac{d}{d u}\left\langle g, \mathbf{1}_{[0, u]}\right\rangle_{\mathfrak{H}}\right|_{u=t}=\left.\frac{d}{d u} \operatorname{Cov}^{\mathbf{Q}}\left(Z(g), Z_{u}\right)\right|_{u=t} \quad \text { exists. }
$$

Moreover,

1. If $Z(g) \mathrm{L}^{2}$-differentiates $Z$ at $t$ under $\mathbf{Q}$, then

$$
D_{\mathbf{P}}^{Z(g)} Z_{t}=\frac{|g|_{\mathfrak{H}}^{2} \mathrm{E}^{\mathbf{P}}\left[Z_{t}-\left\langle\mathbf{1}_{[0, t]}, D \zeta\right\rangle_{\mathfrak{H}} \mid Z(g)\right]}{Z(g)\left\langle g, \mathbf{1}_{[0, t]}\right\rangle_{\mathfrak{H}}} D_{\mathbf{Q}}^{Z(g)} Z_{t}+\mathrm{E}^{\mathbf{P}}\left[\mu_{t} \mid Z(g)\right] .
$$

2. If $Z(g)$ does not $\mathrm{L}^{2}$-differentiate $Z$ at $t$ under $\mathbf{Q}$, then $\mathscr{H} \subset \sigma\{Z(g)\}$ differentiates $Z$ at $t$ with respect to $\mathbf{P}$ if, and only if,

$$
\mathrm{E}^{\mathbf{P}}\left[Z_{t}-\left\langle\mathbf{1}_{[0, t]}, D \zeta\right\rangle_{\mathfrak{H}} \mid \mathscr{H}\right]=0 .
$$

In this case, $D_{\mathbf{P}}^{\mathscr{H}} Z_{t}=\mathrm{E}^{\mathbf{P}}\left[\mu_{t} \mid \mathscr{H}\right]$. 
Remark. Since $Z$ is Gaussian under Q, Corollary 1 implies that $Z(g)$ is not differentiating for $Z$ at $t$ w.r.t. $\mathbf{Q}$ if, and only if, $Z(g)$ is really not differentiating w.r.t. Q. Point 2 in Theorem 2 shows that this double implication does not hold, in general, under the equivalent probability $\mathbf{P}$. Indeed, even if $Z(g)$ does not differentiate $Z$ under $\mathbf{P}$ (and therefore under $\mathbf{Q}$ ), one may have that there exists a differentiating $\mathscr{H} \subset \sigma\{Z(g)\}$ such that $D_{\mathbf{P}}^{\mathscr{H}} Z_{t}$ is non-deterministic. Observe, however, that $D_{\mathbf{P}}^{\mathscr{H}} Z_{t}$ is forced to have the particular form $D_{\mathbf{P}}^{\mathscr{H}} Z_{t}=\mathrm{E}^{\mathbf{P}}\left[\mu_{t} \mid \mathscr{H}\right]$.

Proof: Let $\xi \in \mathrm{L}^{2}(\mathbf{P}) \cap \mathrm{L}^{2}(\mathbf{Q})$ and $A \in \mathscr{G} \subset \mathscr{F}$. The relation

$$
\int_{A} \xi d \mathbf{P}=\int_{A} \mathrm{E}^{\mathbf{P}}[\xi \mid \mathscr{G}] d \mathbf{P}
$$

implies

$$
\begin{aligned}
\int_{A} \mathrm{E}^{\mathbf{Q}}\left[\xi \eta^{-1} \mid \mathscr{G}\right] d \mathbf{Q} & =\int_{A} \xi \eta^{-1} d \mathbf{Q}=\int_{A} \mathrm{E}^{\mathbf{P}}[\xi \mid \mathscr{G}] \eta^{-1} d \mathbf{Q} \\
& =\int_{A} \mathrm{E}^{\mathbf{P}}[\xi \mid \mathscr{G}] \mathrm{E}^{\mathbf{Q}}\left[\eta^{-1} \mid \mathscr{G}\right] d \mathbf{Q} .
\end{aligned}
$$

Thus:

$$
\mathrm{E}^{\mathbf{P}}[\xi \mid \mathscr{G}]=\frac{\mathrm{E}^{\mathbf{Q}}\left[\xi \eta^{-1} \mid \mathscr{G}\right]}{\mathrm{E}^{\mathbf{Q}}\left[\eta^{-1} \mid \mathscr{G}\right]}
$$

from which we deduce that the study of $\mathrm{E}^{\mathbf{P}}\left[\Delta_{h} Z_{t} \mid Z(g)\right]$ can be reduced to that of $\mathrm{E}^{\mathbf{Q}}\left[\eta^{-1} \Delta_{h} Z_{t} \mid Z(g)\right]$. Let $\phi \in \mathcal{C}_{b}^{1}(\mathbb{R})$. We have

$$
\begin{aligned}
\mathrm{E}^{\mathbf{Q}}\left[\left(Z_{t+h}-Z_{t}\right) \eta^{-1} \phi(Z(g))\right]= & \mathrm{E}^{\mathbf{Q}}\left[\left\langle\mathbf{1}_{[t, t+h]}, D\left(\eta^{-1} \phi(Z(g))\right)\right\rangle_{\mathfrak{H}}\right] \\
= & \mathrm{E}^{\mathbf{Q}}\left[\phi(Z(g)) \eta^{-1}\left\langle\mathbf{1}_{[t, t+h]}, D \zeta\right\rangle_{\mathfrak{H}}\right] \\
& +\left\langle\mathbf{1}_{[t, t+h]}, g\right\rangle_{\mathfrak{H}} \mathrm{E}^{\mathbf{Q}}\left[\eta^{-1} \phi^{\prime}(Z(g))\right] .
\end{aligned}
$$

By using an analogous decomposition for $\mathrm{E}^{\mathbf{Q}}\left[Z_{t} \eta^{-1} \phi(Z(g))\right]$, we can also write:

$$
\mathrm{E}^{\mathbf{Q}}\left[\eta^{-1} \phi^{\prime}(Z(g))\right]=\frac{\mathrm{E}^{\mathbf{Q}}\left[\left(Z_{t}-\left\langle\mathbf{1}_{[0, t]}, D \zeta\right\rangle_{\mathfrak{H}}\right) \eta^{-1} \phi(Z(g))\right]}{\left\langle\mathbf{1}_{[0, t]}, g\right\rangle_{\mathfrak{H}}} .
$$

Therefore, $\mathrm{E}^{\mathbf{Q}}\left[\eta^{-1} \Delta_{h} Z_{t} \mid Z(g)\right]$ is equal to

$$
\mathrm{E}^{\mathbf{Q}}\left[\left(Z_{t}-\left\langle\mathbf{1}_{[0, t]}, D \zeta\right\rangle_{\mathfrak{H}}\right) \eta^{-1} \mid Z(g)\right] \frac{\left\langle\mathbf{1}_{[t, t+h]}, g\right\rangle_{\mathfrak{H}}}{h\left\langle\mathbf{1}_{[0, t]}, g\right\rangle_{\mathfrak{H}}}+h^{-1}\left\langle\mathbf{1}_{[t, t+h]}, \mathrm{E}^{\mathbf{Q}}\left[\eta^{-1} D \zeta \mid Z(g)\right]\right\rangle_{\mathfrak{H}}
$$


whereas $\mathrm{E}^{\mathbf{P}}\left[\Delta_{h} Z_{t} \mid Z(g)\right]$ equals the following expression:

$$
\mathrm{E}^{\mathbf{P}}\left[Z_{t}-\left\langle\mathbf{1}_{[0, t]}, D \zeta\right\rangle_{\mathfrak{H}} \mid Z(g)\right] \frac{\left\langle\mathbf{1}_{[t, t+h]}, g\right\rangle_{\mathfrak{H}}}{h\left\langle\mathbf{1}_{[0, t]}, g\right\rangle_{\mathfrak{H}}}+h^{-1}\left\langle\mathbf{1}_{[t, t+h]}, \mathrm{E}^{\mathbf{P}}[D \zeta \mid Z(g)]\right\rangle_{\mathfrak{H}} .
$$

Now, by assumption (12) and thanks to Proposition 11, we have that

$$
\lim _{h \rightarrow 0} h^{-1}\left\langle\mathbf{1}_{[t, t+h]}, \mathrm{E}^{\mathbf{P}}[D \zeta \mid Z(g)]\right\rangle_{\mathfrak{H}}=\mathrm{E}^{\mathbf{P}}\left[\mu_{t} \mid Z(g)\right] \text { in the } \mathrm{L}^{2} \text { topology }
$$

Note moreover that $\mathbf{P}\left(\mathrm{E}^{\mathbf{P}}\left[Z_{t}-\left\langle\mathbf{1}_{[0, t]}, D \zeta\right\rangle_{\mathfrak{H}} \mid Z(g)\right]=0\right)<1$. Indeed, if it was not the case, one would have ( $\delta$ stands for the Skorohod integral)

$$
\begin{aligned}
0 & =\mathrm{E}^{\mathbf{Q}}\left[\left(Z_{t} \eta^{-1}-\left\langle\mathbf{1}_{[0, t]}, D \eta^{-1}\right\rangle_{\mathfrak{H}}\right) Z(g)\right]=\mathrm{E}^{\mathbf{Q}}\left[\delta\left(\mathbf{1}_{[0, t]} \eta^{-1}\right) Z(g)\right] \\
& =\mathrm{E}^{\mathbf{Q}}\left[\eta^{-1}\right]\left\langle\mathbf{1}_{[0, t]}, g\right\rangle_{\mathfrak{H}}=\left\langle\mathbf{1}_{[0, t]}, g\right\rangle_{\mathfrak{H}} \neq 0
\end{aligned}
$$

which is clearly a contradiction. As a consequence, we deduce from (17) that $Z(g)$ $\mathrm{L}^{2}$-differentiates $Z$ at $t$ under $\mathbf{P}$ if, and only if, $\left.\frac{d}{d u}\left\langle g, \mathbf{1}_{[0, u]}\right\rangle_{\mathfrak{H}}\right|_{u=t}$ exists. By Lemma $\mathbb{1}$, this last condition is equivalent to $Z(g)$ being $\mathrm{L}^{2}$-differentiating for $Z$ at $t$ under $\mathbf{Q}$. We can therefore deduce (14) from (17) and (16).

If $\mathscr{H} \subset \sigma\{Z(g)\}$, the projection principle and (17) yield that $\mathrm{E}^{\mathbf{P}}\left[\Delta_{h} Z_{t} \mid \mathscr{H}\right]$ equals

$$
\mathrm{E}^{\mathbf{P}}\left[Z_{t}-\left\langle\mathbf{1}_{[0, t]}, D \zeta\right\rangle_{\mathfrak{H}} \mid \mathscr{H}\right] \frac{\left\langle\mathbf{1}_{[t, t+h]}, g\right\rangle_{\mathfrak{H}}}{h\left\langle\mathbf{1}_{[0, t]}, g\right\rangle_{\mathfrak{H}}}+h^{-1}\left\langle\mathbf{1}_{[t, t+h]}, \mathrm{E}^{\mathbf{P}}[D \zeta \mid \mathscr{H}]\right\rangle_{\mathfrak{H}}
$$

When $\left.\frac{d}{d u}\left\langle\mathbf{1}_{[0, s]}, \mathbf{1}_{[0, u]}\right\rangle_{\mathfrak{H}}\right|_{u=t}$ does not exist, we deduce that $\mathscr{H}$ differentiates $Z$ at $t$ if, and only if, $\mathrm{E}^{\mathbf{P}}\left[Z_{t}-\left\langle\mathbf{1}_{[0, t]}, D \zeta\right\rangle_{\mathfrak{H}} \mid \mathscr{H}\right]=0$. If this condition is verified, we then have $D_{\mathbf{P}}^{\mathscr{H}} Z_{t}=\mathrm{E}^{\mathbf{P}}\left[\mu_{t} \mid \mathscr{H}\right]$, again by Proposition 1 .

As an application of Theorem 2, we shall consider the case where the isonormal process $Z$ in (11) is generated by a fractional Brownian motion of Hurst index $H \in$ $(0,1 / 2) \cup(1 / 2,1)$ (see also [3, Theorem 22], for related results concerning the case $H \in(1 / 2,1))$.

We briefly recall some basic facts about stochastic calculus with respect to a fractional Brownian motion. We refer the reader to [10] for any unexplained notion or result. Let $B=\left(B_{t}\right)_{t \in[0, T]}$ be a fractional Brownian motion with Hurst parameter $H \in(0,1)$, and assume that $B$ is defined on a probability space $(\Omega, \mathscr{F}, \mathbf{P})$. This means that $B$ is a centered Gaussian process with covariance function $\mathrm{E}\left(B_{s} B_{t}\right)=R_{H}(s, t)$ given by

$$
R_{H}(s, t)=\frac{1}{2}\left(t^{2 H}+s^{2 H}-|t-s|^{2 H}\right)
$$


We denote by $\mathcal{E}$ the set of all $\mathbb{R}$-valued step functions on $[0, T]$. Let $\mathfrak{H}$ be the Hilbert space defined as the closure of $\mathcal{E}$ with respect to the scalar product

$$
\left\langle\mathbf{1}_{[0, t]}, g\right\rangle_{\mathfrak{H}}=R_{H}(t, s),
$$

and denote by $|\cdot|_{\mathfrak{H}}$ the associate norm. The mapping $\mathbf{1}_{[0, t]} \mapsto B_{t}$ can be extended to an isometry between $\mathfrak{H}$ and the Gaussian space $\mathcal{H}_{1}(B)$ associated with $B$. We denote this isometry by $\varphi \mapsto B(\varphi)$. Recall that the covariance kernel $R_{H}(t, s)$ introduced in (18) can be written as

$$
R_{H}(t, s)=\int_{0}^{s \wedge t} K_{H}(s, u) K_{H}(t, u) d u,
$$

where $K_{H}(t, s)$ is the square integrable kernel defined, for $s<t$, by

$$
K_{H}(t, s)=\Gamma\left(H+\frac{1}{2}\right)^{-1}(t-s)^{H-\frac{1}{2}} F\left(H-\frac{1}{2}, \frac{1}{2}-H, H+\frac{1}{2}, 1-\frac{t}{s}\right),
$$

where $F(a, b, c, z)$ is the classical Gauss hypergeometric function. By convention, we set $K_{H}(t, s)=0$ if $s \geq t$. We define the operator $\mathcal{K}_{H}$ on $\mathrm{L}^{2}([0, T])$ as

$$
\left(\mathcal{K}_{H} h\right)(t)=\int_{0}^{t} K_{H}(t, s) h(s) d s
$$

Let $\mathcal{K}_{H}^{*}: \mathcal{E} \rightarrow \mathrm{L}^{2}([0, T])$ be the linear operator defined as:

$$
\mathcal{K}_{H}^{*}\left(\mathbf{1}_{[0, t]}\right)=K_{H}(t, \cdot) .
$$

The following equality holds for any $\phi, \psi \in \mathcal{E}$

$$
\langle\phi, \psi\rangle_{\mathfrak{H}}=\left\langle\mathcal{K}_{H}^{*} \phi, \mathcal{K}_{H}^{*} \psi\right\rangle_{\mathrm{L}^{2}([0, T])}=\mathrm{E}(B(\phi) B(\psi)),
$$

implying that $\mathcal{K}_{H}^{*}$ is indeed an isometry between the Hilbert spaces $\mathfrak{H}$ and a closed subspace of $\mathrm{L}^{2}([0, T])$. Now consider the process $W=\left(W_{t}\right)_{t \in[0, T]}$ defined as

$$
W_{t}=B\left(\left(\mathcal{K}_{H}^{*}\right)^{-1}\left(\mathbf{1}_{[0, t]}\right)\right),
$$

and observe that $W$ is a standard Wiener process, and also that the process $B$ has an integral representation of the type

$$
B_{t}=\int_{0}^{t} K_{H}(t, s) d W_{s}
$$

so that, for any $\phi \in \mathfrak{H}$,

$$
B(\phi)=W\left(\mathcal{K}_{H}^{*} \phi\right)
$$


We will also need the fact that the operator $\mathcal{K}_{H}$ can be expressed in terms of fractional integrals as follows:

$$
\begin{array}{ll}
\left(\mathcal{K}_{H} h\right)(s)=I_{0+}^{2 H} s^{\frac{1}{2}-H} I_{0+}^{\frac{1}{2}-H} s^{H-\frac{1}{2}} h(s), & \text { if } H<1 / 2, \\
\left(\mathcal{K}_{H} h\right)(s)=I_{0+}^{1} s^{H-\frac{1}{2}} I_{0+}^{H-\frac{1}{2}} s^{\frac{1}{2}-H} h(s), & \text { if } H>1 / 2,
\end{array}
$$

for every $h \in \mathrm{L}^{2}([0, T])$. Here, $I_{0+}^{\alpha} f$ denotes the left fractional Riemann-Liouville integral of order $\alpha$ of $f$, which is defined by

$$
I_{0+}^{\alpha} f(x)=\frac{1}{\Gamma(\alpha)} \int_{0}^{x}(x-y)^{\alpha-1} f(y) d y .
$$

Let $\Upsilon_{H}$ be the set of the so-called shifted fBm $Z=\left(Z_{t}\right)_{t \in[0, T]}$ defined by

$$
Z_{t}=x_{0}+B_{t}+\int_{0}^{t} b_{s} d s, \quad t \in[0, T]
$$

where $b$ runs over the set of adapted processes (w.r.t. the natural filtration of $B$ ) having integrable trajectories.

We also need to introduce a technical assumption. Define

$$
a_{r}=\left(\mathcal{K}_{H}^{-1} \int_{0}^{\cdot} b_{s} d s\right)(r)
$$

in what follows we shall always assume that

(H1) $a$ is bounded a.s.,

(H2) $\Phi$ defined by $\Phi(s)=\int_{0}^{T} D_{s} a_{r} \delta B_{r}$ exists and belongs in $\mathrm{L}^{2}([0, T])$ a.s..

First, let us consider the case $H>1 / 2$. We suppose moreover that the trajectories of $b$ are a.s. Hölder continuous of order $H-1 / 2+\varepsilon$, for some $\varepsilon>0$. Then, the fractional version of the Girsanov theorem (see [11, Theorem 2]) applies, yielding that $Z$ is a fractional Brownian motion of Hurst parameter $H$ under the new probability $\mathbf{Q}$ defined by $d \mathbf{Q}=\eta d \mathbf{P}$, where

$$
\eta=\exp \left(-\int_{0}^{T}\left(\mathcal{K}_{H}^{-1} \int_{0} b_{r} d r\right)(s) d W_{s}-\frac{1}{2} \int_{0}^{T}\left(\mathcal{K}_{H}^{-1} \int_{0}^{.} b_{r} d r\right)^{2}(s) d s\right) .
$$

We can now state the following extension of Theorem 22 in [3]:

Corollary 2 Let $Z \in \Upsilon_{H}$ with $H>1 / 2$ and $s, t \in(0, T)$. Then $Z_{s} \mathrm{~L}^{2}$-differentiates $Z$ at $t$. 
Proof: The proof of this result relies on Theorem 2. Note also that parts of the arguments rehearsed below are only sketched, since they are analogous to those involved in the proof of [3, Theorem 22]. Let us consider

$$
\zeta=\int_{0}^{T} a_{s} d W_{s}+\frac{1}{2} \int_{0}^{T} a_{s}^{2} d s,
$$

where $a$ is defined according to (23). We shall show that (12) holds. We can compute (see the proof of [3, Theorem 22])

$$
\left\langle\mathbf{1}_{[t, t+h]}, D \zeta\right\rangle_{\mathfrak{H}}=\int_{t}^{t+h} b_{r} d r+\left(\mathcal{K}_{H} \Phi\right)(t+h)-\left(\mathcal{K}_{H} \Phi\right)(t),
$$

where $\Phi(s)=\int_{0}^{T} D_{s} a_{r} \delta B_{r}$, see (H2). Since, in the case where $H>1 / 2, \mathcal{K}_{H} \Phi$ is differentiable at $t$ (see for instance (21)) we deduce that (12) holds. Moreover, one can easily prove that (13) also holds, so that the proof is concluded.

Now we consider the case $H<1 / 2$. We assume moreover that $\int_{0}^{T} b_{r}^{2} d r<+\infty$ a.s.. Then, the fractional version of the Girsanov theorem (see [11, Theorem 2]) holds again, implying that $Z$ is a fractional Brownian motion of Hurst parameter $H$ under the new probability $\mathbf{Q}$ defined by $d \mathbf{Q}=\eta d \mathbf{P}$, with $\eta$ given by (24). Note that, when $H<1 / 2$, we cannot apply Theorem 2, since (12) does not hold in general. The reason is that $\mathcal{K}_{H} \Phi$ is no more differentiable at $t$, see (20). In order to make $h^{-1} \mathrm{E}\left[Z_{t+h}-Z_{t} \mid Z(g)\right]$ converge, we have to replace $h^{-1}$ with $h^{-2 H}$ (we only consider the case where $h>0$ ). This fact is made precise by the following result.

Proposition 2 Let $Z \in \Upsilon_{H}$ with $H<1 / 2$ and $s, t \in(0, T)$. Then,

$$
\lim _{h \downarrow 0} h^{-2 H} \mathrm{E}\left[Z_{t+h}-Z_{t} \mid Z_{s}\right] \text { exists in the } \mathrm{L}^{2} \text {-topology. }
$$

Proof: We go back to the proof of Corollary 2, with special attention to relation (25). 
By setting $\phi(s)=s^{\frac{1}{2}-H} I_{0+}^{\frac{1}{2}-H} s^{H-\frac{1}{2}} \Phi(s)$, we have

$$
\begin{aligned}
\mathcal{K}_{H} \Phi(t+h)-\mathcal{K}_{H} \Phi(t)= & I_{0+}^{2 H} \phi(t+h)-I_{0+}^{2 H} \phi(t) \\
= & \frac{1}{\Gamma(2 H)} \int_{0}^{t}\left((t+h-y)^{2 H-1}-(t-y)^{2 H-1}\right) \phi(y) d y \\
& \quad+\frac{1}{\Gamma(2 H)} \int_{t}^{t+h}(t+h-y)^{2 H-1} \phi(y) d y \\
= & \frac{1}{\Gamma(2 H)} \int_{0}^{t}\left((y+h)^{2 H-1}-y^{2 H-1}\right) \phi(t-y) d y \\
& \quad+\frac{1}{\Gamma(2 H)} \int_{0}^{h} y^{2 H-1} \phi(t+h-y) d y \\
= & \frac{h^{2 H}}{\Gamma(2 H)} \int_{0}^{t / h}\left((y+1)^{2 H-1}-y^{2 H-1}\right) \phi(t-h y) d y \\
& +\frac{h^{2 H}}{\Gamma(2 H)} \int_{0}^{1} y^{2 H-1} \phi(t+h-h y) d y .
\end{aligned}
$$

We deduce that

$$
h^{-2 H}\left(\mathcal{K}_{H} \Phi(t+h)-\mathcal{K}_{H} \Phi(t)\right) \longrightarrow c_{H} \phi(t), \text { as } h \rightarrow 0,
$$

where

$$
c_{H}=\frac{1}{\Gamma(2 H)} \int_{0}^{+\infty}\left((y+1)^{2 H-1}-y^{2 H-1}\right) d y+\frac{1}{\Gamma(2 H)} \int_{0}^{1} y^{2 H-1} d y<+\infty .
$$

Thus, by using the notations adopted in (the proof of) Theorem 2, one deduces an analogue of (12), obtained by replacing $h^{-1}$ with $h^{-2 H}$, that is:

$$
\tilde{\mu}_{t} \triangleq \lim _{h \rightarrow 0} h^{-2 H}\left\langle\mathbf{1}_{[t, t+h]}, D \zeta\right\rangle_{\mathfrak{H}} \quad \text { exists in the } \mathrm{L}^{2} \text { topology. }
$$

Moreover, it is easily shown that $\lim _{h \rightarrow 0} h^{-2 H}\left\langle\mathbf{1}_{[t, t+h]}, \mathbf{1}_{[0, s]}\right\rangle_{\mathfrak{H}}$ exists. By using (17), we obtain the desired conclusion.

\section{Differentiating collections of $\sigma$-fields and the as- sociated differentiated process}

In this section, we work on a complete probability space $(\Omega, \mathscr{F}, \mathbf{P})$, and we denote by $\mathscr{B}_{(0, T)}$ the Borel $\sigma$-field of $(0, T)$. In the previous sections, we have studied the 
properties of those $\sigma$-field that are differentiating for some processes at a fixed time $t$. We will now concentrate on collections of differentiating $\sigma$-fields indexed by the whole interval $(0, T)$.

Definition 4 We say that a collection $\left(\mathscr{A}^{t}\right)_{t \in(0, T)}$ of $\sigma$-fields $\tau$-differentiates $Z$ if, for any $t \in(0, T), \mathscr{A}^{t} \tau$-differentiates $Z$ at $t$.

A differentiating collection of $\sigma$-fields need not be a filtration (see $e . g$. section 5 in [3]). Nevertheless, we can associate to each $\tau$-differentiating collection $\mathfrak{A}=$ $\left(\mathscr{A}^{t}\right)_{t \in(0, T)}$ for $Z$ a filtration $\mathcal{A}=\left(\mathscr{A}_{t}\right)_{t \in(0, T)}$, obtained by setting:

$$
\mathscr{A}_{t}=\bigvee_{0<s \leqslant t} \mathscr{A}^{t}, \quad t \in(0, T) .
$$

The collection of r.v. $\left(D^{\mathscr{A}^{t}} Z_{t}\right)_{t \in(0, T)}$ is a $\mathcal{A}$-adapted process $\llbracket 12$, Definition 27.1], in the sense that for all $t \in(0, T), D^{\mathscr{A}^{t}} Z_{t}$ is $\mathscr{A}_{t}$-measurable. We call it the differentiated process of $Z$ w.r.t. $\mathfrak{A}$, and we denote it by $D^{\mathfrak{A}} Z$.

In order to use such a process in stochastic analysis, one should know whether it admits a measurable version, that is, whether there exists a process $Y$ which is $\mathscr{B}_{(0, T)} \otimes \mathscr{F}$-measurable and such that for all $t, Y_{t}=D^{\mathscr{A}^{t}} Z_{t}$ a.s.. Our aim in this section is to obtain a sufficient condition for the existence of a measurable version. To this end, we introduce the following

Definition 5 Let $\mathfrak{A}=\left(\mathscr{A}^{t}\right)_{t}$ be a collection of $\sigma$-fields and $Z$ be a measurable stochastic process. We say that $\mathfrak{A}$ is regular for $Z$ if for all $n \in \mathbb{N}, i \in\{1, \cdots, n\}, t_{i} \in[0, T]$, $\phi_{i} \in C_{0}^{\infty}\left(\mathbb{R}^{d}\right)$, the process

$$
t \mapsto \mathrm{E}\left[\phi_{1}\left(Z_{t_{1}}\right) \cdots \phi_{n}\left(Z_{t_{n}}\right) \mid \mathscr{A}^{t}\right]
$$

has a measurable version.

If $\mathfrak{A}$ is a filtration, then $\mathfrak{A}$ is regular for any process. For Gaussian processes and most of drifted Gaussian processes $X$, the collection $\mathfrak{A}=\left(\sigma\left\{X_{t}\right\}\right)_{t}$ is a regular collection for $X$.

The next result shows that, under the regularity condition defined above, a measurable version of the differentiated process exists. This follows from one of Doob's most celebrated theorems (see e.g. [四, Theorem 30 p.158]). 
Theorem 3 Let $X$ be a $\mathscr{B}_{(0, T)} \otimes \mathscr{F}$-measurable stochastic process defined on a complete probability space $(\Omega, \mathscr{F}, \mathbf{P})$, and assume that $\mathscr{F}=\sigma\{X\}$. Let $\mathfrak{A}$ be a regular $L^{1}$-differentiating collection for $X$. Then, there exists a measurable version of the differentiated process $D^{\mathfrak{A}} X$. This version is also adapted to the filtration generated by $\mathfrak{A}$.

Proof: Fix $\varepsilon>0$, and let $\left(h_{k}\right)$ be a sequence converging to 0 and $Z^{k}$ be the process defined by

$$
Z_{t}^{k}=\frac{X_{t+h_{k}}-X_{t}}{h_{k}}
$$

Since $X$ is measurable, so is the process $Z$. Then, by [4, Theorem 30 p.158], there exist elementary processes $U_{t}^{n_{k}}$ such that, for all $t \in(0, T)$ and every $k, \mathrm{E}\left|Z_{t}^{k}-U_{t}^{n_{k}}\right|<\varepsilon / 2$. These elementary processes have the form:

$$
U_{t}^{n_{k}}=\sum_{i} \mathbf{1}_{A_{i}^{n_{k}}}(t) H_{i}^{n_{k}}
$$

where $\left(A_{i}^{n_{k}}\right)_{i}$ is a finite partition of $(0, T)$ and $H_{i}^{n_{k}}$ are $\mathscr{F}$-measurable random variables. We have

$$
\mathrm{E}\left[U_{t}^{n_{k}} \mid \mathscr{A}^{t}\right]=\sum_{i} \mathbf{1}_{A_{i}^{n_{k}}}(t) \mathrm{E}\left[H_{i}^{n_{k}} \mid \mathscr{A}^{t}\right]
$$

Since cylindrical functionals of $X$ are dense in $L^{1}(\Omega, \mathscr{F})$, we deduce from the regularity condition that the processes $t \mapsto \mathrm{E}\left[H_{i}^{n_{k}} \mid \mathscr{A}^{t}\right]$ admits a $\mathscr{B}_{(0, T)} \otimes \mathscr{F}$-measurable modification and also, by linearity, the same conclusion holds for the process $t \mapsto \mathrm{E}\left[U_{t}^{n_{k}} \mid \mathscr{A}^{t}\right]$. Moreover,

$$
\mathrm{E}\left|\mathrm{E}\left[Z_{t}^{k} \mid \mathscr{A}^{t}\right]-\mathrm{E}\left[U_{t}^{n_{k}} \mid \mathscr{A}^{t}\right]\right|<\varepsilon / 2
$$

Since $\mathfrak{A}$ is a $L^{1}$-differentiating collection for $X$, we deduce that there exists $k$ such that

$$
\mathrm{E}\left|D^{\mathscr{A}^{t}} X_{t}-\mathrm{E}\left[Z_{t}^{k} \mid \mathscr{A}^{t}\right]\right|<\varepsilon / 2
$$

and therefore $\mathrm{E}\left|D^{\mathscr{A}^{t}} X_{t}-E\left[U_{t}^{n_{k}} \mid \mathscr{A}^{t}\right]\right|<\varepsilon$ for every $t$.

We now deduce that the map $t \mapsto\left[D^{\mathscr{A}^{t}} X_{t}\right]$ is measurable, where [.] denotes the class of a process in $L^{1}(\Omega)$ reduced by null sets. Indeed, it is the limit in the Banach space $L^{1}(\Omega)$ (when $k$ goes to infinity) of the measurable map $t \mapsto \mathrm{E}\left[U_{t}^{n_{k}} \mid \mathscr{A}^{t}\right]$. Since $L^{1}(\Omega)$ is separable, we again deduce from $\left[4\right.$, Theorem 30 p.158] that $D^{\mathfrak{A}} X$ admits a measurable modification. 


\section{Embedded differential equations}

The last section of the paper is devoted to the outline of a general framework for stochastic embedding problems (introduced in [2]) related to ordinary differential equations. As we will see, this notion involves the stochastic derivative operators that we have defined and studied in the previous sections. Roughly speaking, the aim of a stochastic embedding procedure is to write a "stochastic equation" which admits both stochastic and deterministic solutions, in such a way that the deterministic solutions also satisfy a fixed ordinary differential equation (see [2]). It follows that the embedded stochastic equation is a genuine extension of the underlying ordinary differential equation to a stochastic framework.

\subsection{General setting}

Let $\chi: \mathbb{R}^{d} \rightarrow \mathbb{R}^{d}\left(d \in \mathbb{N}^{*}\right)$ be a smooth vector field. Consider the ordinary differential equation:

$$
\frac{d x}{d t}(t)=\chi(x(t)), \quad t \in[0, T]
$$

Let $\Lambda$ be a set of measurable stochastic processes $X: \Omega \times[0, T] \rightarrow \mathbb{R}^{d}$, where $(\Omega, \mathscr{F}, \mathbf{P})$ is a fixed probability space. In order to distinguish two different kinds of families of $\sigma$-fields, we shall adopt the following notation: (i) the symbol $\mathfrak{A}_{0}=$ $\left(\mathscr{A}_{0}^{t}\right)_{t \in[0, T]}$ denotes a collection of $\sigma$-fields whose definition does not depend on the choice of $X$ in the class $\Lambda$, and (ii) $\mathfrak{A}=\left(\mathscr{A}_{X}^{t}\right)_{X \in \Lambda, t \in[0, T]}$ indicates a generic family of $\sigma$-fields such that, for every $t \in[0, T]$ and every $X \in \Lambda, \mathscr{A}_{X}^{t} \subset \mathscr{P}_{T}^{X}$. We introduce the following natural assumption:

(T) $\Lambda$ contains all the deterministic differentiable functions $f:[0, T] \rightarrow \mathbb{R}^{d}$ (viewed as deterministic stochastic processes).

We now fix a topology $\tau$, and describe two stochastic embedded equations associated with (26).

Definition 6 Fix a class of stochastic processes $\Lambda$ on $(\Omega, \mathscr{F}, \mathbf{P})$, verifying assumption $(T)$.

(a) Given a family $\mathfrak{A}_{0}=\left(\mathscr{A}_{0}^{t}\right)_{t \in[0, T]}$ of $\sigma$-fields, we say that the equation

$$
X \in \Lambda, \quad D^{\mathscr{A}_{0}^{t}} X_{t}=\chi\left(X_{t}\right) \quad \text { for every } t \in[0, T],
$$

is the strong stochastic embedding in $\Lambda$ of the ODE (20) w.r.t. $\mathfrak{A}_{0}$.

(b) Given a family $\mathfrak{A}=\left(\mathscr{A}_{X}^{t}\right)_{X \in \Lambda, t \in[0, T]}$ of $\sigma$-fields such that for all $X \in \Lambda$ and $t \in[0, T], \mathscr{A}_{X}^{t} \subset \mathscr{P}_{T}^{X}$, we say that the equation

$$
X \in \Lambda, \quad D^{\mathscr{A}_{X}^{t}} X_{t}=\chi\left(X_{t}\right) \quad \text { for every } t \in[0, T],
$$


is the weak stochastic embedding in $\Lambda$ of the $O D E(\sqrt[20]{)}$ w.r.t. $\mathfrak{A}$.

(c) A solution of (27) (resp. (28)) is a stochastic process $X \in \Lambda$ such that: (c1 ) the process $D^{\mathscr{A}_{0}^{t}} X_{t}$ (resp. $D^{\mathscr{A}_{X}^{t}} X_{t}$ ) admits a jointly measurable version, and (c-2) the equation $D^{\mathscr{A}_{0}^{t}} X_{t}=\chi\left(X_{t}\right)$ (resp. $D^{\mathscr{A}_{X}^{t}} X_{t}=\chi\left(X_{t}\right)$ ) is verified for every $t \in[0, T]$.

Note that a solution of (26) is always a solution of (27) or (28). Observe also that if one wants to obtain "genuinely stochastic" solutions of (26) (i.e. non deterministic), the previous definition implicitly imposes some restrictions on the class $\Lambda$. Namely, if $X \in \Lambda$ is a solution of (27) (resp. (28)), then for any $t \in[0, T]$, $\mathscr{A}_{0}^{t}$ (resp. $\mathscr{A}_{X}^{t}$ ) is differentiating for $X$ at $t$ with respect to the topology $\tau$ and the random variable $\chi\left(X_{t}\right)$ is $\mathscr{A}_{0}^{t}$-measurable (resp. $\mathscr{A}_{X}^{t}$-measurable) for every $t$. As an example, let $\Gamma$ be the set of all processes $X$ with the form:

$$
X_{t}=X_{0}+\sigma B_{t}+\int_{0}^{t} b_{r} d r, \quad t \in[0, T]
$$

where $\sigma \in \mathbb{R}, B$ is a $\mathrm{fBm}$ of Hurst index $H \in(0,1)$, and $b$ runs over the set of adapted processes (w.r.t. the natural filtration of $B^{H}$ ) having a.s. integrable trajectories. Suppose that we seek for solutions with $\sigma \neq 0$ of the weak stochastic embedding of (26) given by

$$
X \in \Gamma, \quad D^{\sigma\left\{X_{t}\right\}} X_{t}=\chi\left(X_{t}\right),
$$

Then, Corollary 2 and Proposition 2 imply that such solutions must necessarily be driven by a $\mathrm{fBm}$ of Hurst index $H>1 / 2$.

Stochastic embedded equations may be useful in the following framework. Suppose that a physical system is described by (26), and that we want to enhance this deterministic mathematical model in order to take into account some "stochastic phenomenon" perturbing the system. Then, the embedded equations (27) or (28) may be the key to define a stochastic model in a very coherent way, in the sense that every stochastic process satisfying (27) or (28) is also constrained by the physical laws (i.e. the ODE (26)) defining the original deterministic description of the system.

\subsection{A first example}

Consider the set $\Lambda$ of all continuous processes defined on the probability space $(\Omega, \mathscr{F}, \mathbf{P})$, as well as the "constant" collection of $\sigma$-fields $\left(\mathscr{F}_{t}\right)_{t \in[0, T]}$ such that $\mathscr{F}_{t}=\mathscr{F}$ for every $t$. Since the stochastic derivative w.r.t. $\mathscr{F}$ coincides with the usual pathwise derivative, the embedding problem

$$
D^{\mathscr{F}} X_{t}=\chi\left(X_{t}\right), \quad t \in[0, T]
$$


has a unique strong solution for a given initial condition (deterministic or random). Note that in this example the embedded differential equation produces no other solution than those given by (26).

\subsection{A more interesting example}

Let $W$ be a Wiener process on $[0, T]$ and consider the set $\Lambda$ of deterministic processes and of all stochastic processes that can be expressed in terms of multiple stochastic integrals with respect to $W$. More precisely, denote by $\Lambda_{W}$ the set of processes $u \in \mathrm{L}^{2}\left(\Omega, \mathrm{L}^{2}([0, T])\right)$ such that, for every $t \in[0, T]$,

$$
u_{t}=\sum_{n \geq 0} J_{n}\left(f_{n}(\cdot, t)\right)
$$

where, for any $t \in[0, T]$, the $f_{n}(\cdot, t)$ 's verify:

$$
\sum_{n \geq 0}\left(\left\|f_{n}(\cdot, t)\right\|_{\mathrm{L}^{2}\left(\Delta_{n}[0, T]\right)}^{2}+\left\|\frac{\partial f_{n}}{\partial t}(\cdot, t)\right\|_{\mathrm{L}^{2}\left(\Delta_{n}[0, t]\right)}^{2}\right)<+\infty .
$$

Here

$$
\Delta_{n}[0, T]=\left\{\left(s_{1}, \ldots, s_{n}\right) \in \mathbb{R}_{+}^{n}: 0 \leq s_{n} \leq \ldots \leq s_{1} \leq T\right\}
$$

and, for $g \in \mathrm{L}^{2}\left(\Delta_{n}[0, T]\right)$,

$$
J_{n}(g)=\int_{\Delta_{n}[0, T]} g d W=\int_{0}^{T} d W_{s_{1}} \int_{0}^{s_{1}} d W_{s_{2}} \ldots \int_{0}^{s_{n-1}} d W_{s_{n}} g\left(s_{1}, \ldots, s_{n}\right) .
$$

On $\Lambda_{W}$, we can consider stochastic derivatives of Nelson type (i.e. w.r.t. a fixed filtration $[8]$ ):

Lemma 2 Fix $t \in] 0, T\left[\right.$ and let $\mathscr{P}_{t}$ be the past before $t$, that is the $\sigma$-field generated by $\left\{W_{s}, 0 \leq s \leq t\right\}$. If $u \in \Lambda_{W}$ then $D^{\mathscr{P}_{t}} u_{t}$ exists and it is given by

$$
D^{\mathscr{P}_{t}} u_{t}=\sum_{n \geq 0} J_{n}\left(\frac{\partial f_{n}}{\partial t}(\cdot, t) \mathbf{1}_{\Delta_{n}[0, t]}\right) \text { in the } \mathrm{L}^{2} \text { sense. }
$$

Proof: Obvious by projection.

As an example, consider the case where $\chi$ is given by $\chi(x)=a x+b$ with $a, b \in \mathbb{R}$. In other words, we want to solve the strong embedding

$$
X \in \Lambda, \quad D^{\mathscr{P} t} X_{t}=a X_{t}+b, \quad t \in[0, T]
$$


in the class $\Lambda_{W}$. It is easy to see that if $X \in \Lambda_{W}$, then $X$ satisfies (33) if, and only if, the kernels in its chaotic expansion satisfy

$$
\left.\frac{\partial f_{n}}{\partial t}(\cdot, t) \mathbf{1}_{\Delta_{n}[0, t]}(\cdot)=a f_{n}(\cdot, t), \quad t \in\right] 0, T[
$$

for any $n \in \mathbb{N}^{*}$ and

$$
\left.f_{0}^{\prime}(t)=a f_{0}(t)+b, \quad t \in\right] 0, T[.
$$

We deduce that $X \in \Lambda_{W}$ solves strongly (33) if, and only if, there exists a sequence $\left(c_{n}\right)_{n \in \mathbb{N}}$ of functions from $\Delta_{n}[0, T]$ to $\mathbb{R}$ such that

$$
f_{n}(\cdot, t)=c_{n}(\cdot) \mathrm{e}^{a t} \mathbf{1}_{\Delta_{n}[0, t]}(\cdot), \quad t \in[0, T]
$$

for every $n \in \mathbb{N}^{*}$, and

$$
f_{0}(t)=c_{0} \mathrm{e}^{a t}-b / a, \quad t \in[0, T]
$$

Several properties of embedded stochastic equations will be investigated in a separate paper. For instance, we will be interested in establishing conditions ensuring that the solution of an embedded equation is Markovian. Also, we will explore embedded stochastic equations that are obtained from ordinary equations of order greater than one.

\section{References}

[1] E. Carlen (2006). Stochastic mechanics: a look back and a look ahead. In: Diffusion, Quantum Theory and Radically Elementary Mathematics. Princeton University Press, Princeton, NJ, 117-139

[2] J. Cresson and S. Darses (2006). Plongement stochastique des systèmes lagrangiens. C.R. Acad. Sci. Paris Ser. I 342 (5), 333-336.

[3] S. Darses and I. Nourdin (2006). Stochastic derivatives for fractional diffusions. To appear in the Annals of Probability.

[4] C. Dellacherie and P.-A. Meyer (1975). Probabilits et potentiel. (French) Chapitres I IV. dition entirement refondue. Publications de l'Institut de Mathmatique de l'Universit de Strasbourg, No. XV. Actualits Scientifiques et Industrielles, No. 1372. Hermann, Paris. 
[5] H. Föllmer (1984). Time reversal on Wiener space. Stochastic processes - mathematics and physics (Bielefeld). Lecture Notes in Math. 1158, 119-129.

[6] K. Itô and M. Nisio (1968). On the convergence of sums of independent Banach space valued random variables. Osaka J. Math. 5, 35-48.

[7] T. T. Kadota (1967). Differentiation of Karhunen-Loève expansion and application to optimum reception of sure signals in noise. IEEE Transactions on Information Theory, 13(2), 255-260.

[8] E. Nelson (1966): Dynamical theory of Brownian motion. Princeton University Press. (second edition available online at http://www.math.princeton.edu/ nelson/books/bmotion.pdf).

[9] D. Nualart (2006). The Malliavin Calculus and Related Topics. Second Edition. Springer-Verlag. Berlin Heidelberg New York.

[10] D. Nualart (2003). Stochastic calculus with respect to the fractional Brownian motion and applications. Contemporary Mathematics 336, 3-39.

[11] D. Nualart and Y. Ouknine (2002). Regularization of differential equations by fractional noise. Stochastic Proc. Appl. 102, 103-116.

[12] L.C.G. Rogers and D. Williams (1994). Diffusions, Markov processes, and martingales. Vol. 1. Foundations. Cambridge Mathematical Library. Cambridge University Press. 